\title{
Tonal signal detection in passive sonar systems using atomic norm minimization
}

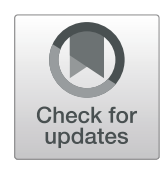

\author{
Jinhong Kim ${ }^{1}$ Junhan Kim ${ }^{1}$, Luong Trung Nguyen ${ }^{1}$, Byonghyo Shim ${ }^{1}$ and Wooyoung Hong ${ }^{2 *}$
}

\begin{abstract}
Frequency estimation of a tonal signal in passive sonar systems is crucial to the identification of the marine object. In the conventional techniques, a basis mismatch error caused by the discretization of the frequency domain is unavoidable, resulting in a severe degradation of the object detection quality. To overcome the basis mismatch error, we propose a tonal frequency estimation technique in the continuous frequency domain. Towards this end, we formulate the frequency estimation problem as an atomic norm minimization problem. From the numerical experiments, we show that the proposed technique is effective in identifying the tonal frequency components of marine objects.
\end{abstract}

Keywords: Passive sonar, Tonal signal detection, Atomic norm minimization

\section{Introduction}

Recently, passive sonar has received much attention as a means to detect acoustic signals radiated from marine objects (e.g., submarines, ships, and marine animals) [1-4]. The passive sonar system is preferred since an intentional signal transmission is unnecessary.

Roughly speaking, the signal detected by the passive sonar consists of four components: tonal signal, propeller noise, hydrodynamic noise, and ambient noise [5] (see Fig. 1). Among them, the tonal signal, the sum of sinusoidal tones occurred by a marine object [6], has received special attention as a tool to identify the marine object. Since each object can be characterized by the frequency dependent features, accurate identification of the frequency components in a tonal signal is of great importance for the target object detection.

Traditionally, an approach to use the discrete Fourier transform (DFT) has been popularly employed for implementation simplicity [7]. One well-known drawback of this approach is that the frequency resolution (spacing between adjacent points in the frequency domain) is limited. In fact, since the frequency resolution of the DFTbased approach is inversely proportional to the observation interval, a small observation time might cause a basis

\footnotetext{
*Correspondence: wyhong@sejong.ac.kr

${ }^{2}$ Dept. of Defense Systems Engineering, Sejong Univ., 209 Neungdong-ro,

Gunja-dong, Gwangjin-gu, 05006 Seoul, South Korea

Full list of author information is available at the end of the article
}

mismatch in the frequency domain, resulting in a severe degradation of the object detection quality. To overcome the shortcoming, various spectral estimation techniques have been proposed over the years [8-10]. Representative techniques include root multiple signal classification (root-MUSIC) [8], estimation of signal parameters via rotational invariance techniques (ESPRIT) [9], and matrix pencil algorithm [10]. These approaches estimate the frequency components using the eigenvectors in the signal subspace. In obtaining the signal subspace, the correlation matrix constructed from the large number of sampled data is required. In addition, the number of frequency components should be known a priori or estimated in advance, to determine the number of eigenvectors used in the frequency estimation.

As an alternative approach, compressed sensing (CS)based techniques have been employed in identifying the tonal frequency components $[11,12]$. It is now wellknown from the theory of CS that a high-dimensional signal can be recovered from a relatively small number of measurements as long as the desired signal is sparse (i.e., the number of nonzero elements in the signal is small). The main observation behind the CS-based techniques is that a tonal signal consists of a small number of frequency components. Motivated from this observation, the CS-based techniques construct a discretized frequency basis and then identify the basis components representing the tonal signal. While the CS-based approaches are 


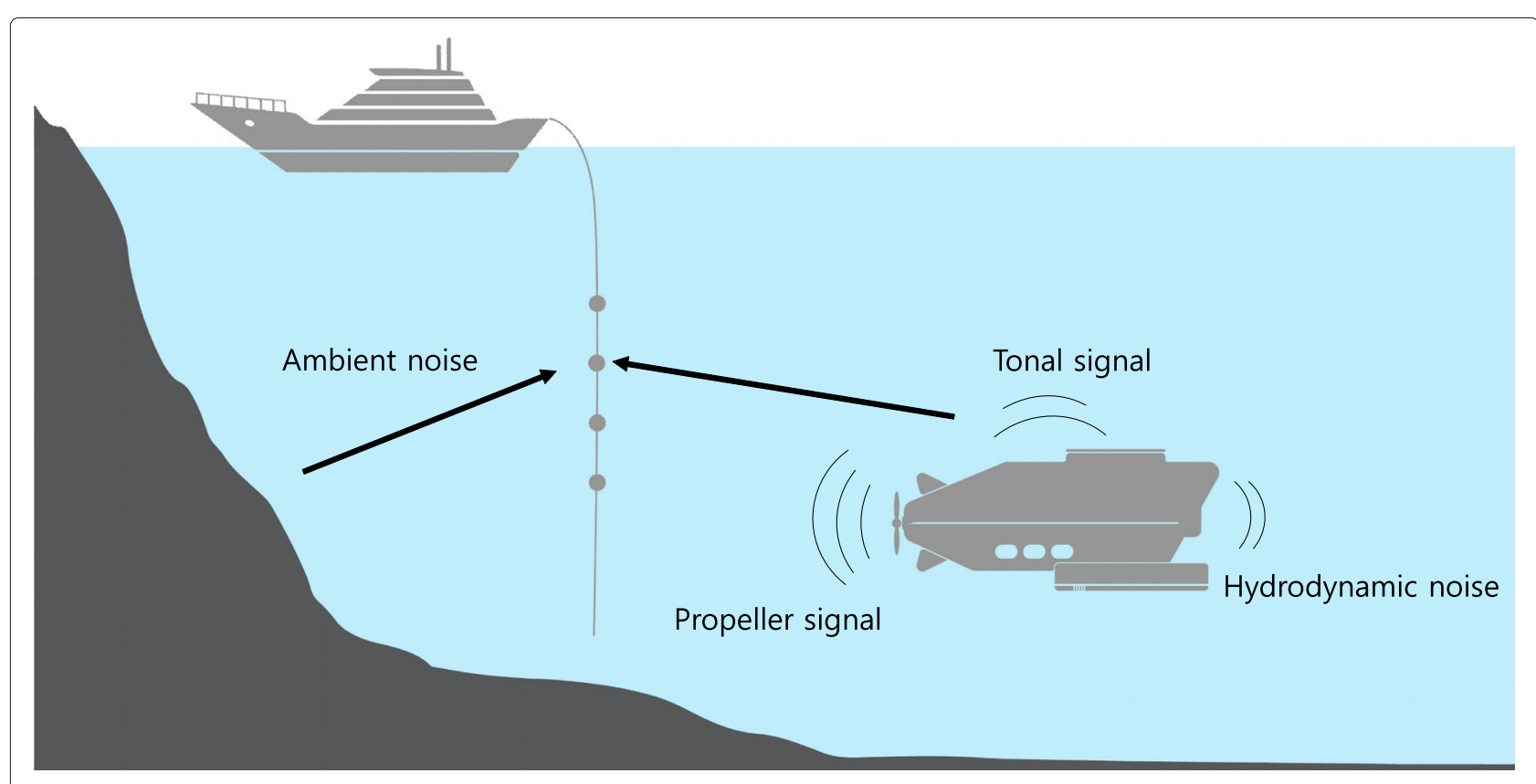

Fig. 1 Types of signal in underwater environments

effective in terms of the computational complexity and the accuracy of the estimated frequency components, a basis mismatch error caused by the discretization of the frequency axis is unavoidable.

The primary goal of this paper is to put forth a new approach to estimate the frequency components of the tonal signal. The key idea of the proposed approach is to formulate the tonal frequency estimation problem as an atomic norm minimization (ANM) problem [13-15]. In a nutshell, ANM can be thought as an extension of the CS technique in which the sensing matrix is allowed to have an infinite number of columns (atoms). One clear benefit of using the ANM technique is that one can avoid the basis mismatch error caused by the discretization of the frequency domain, since ANM identifies the desired frequency components in a continuous domain.

From the simulations using synthetic and real experimental data, we demonstrate that the proposed detection technique is effective in identifying the tonal frequency components. In particular, when the number of frequency components is larger than 15 , the proposed technique shows roughly two times larger success probability than those of the conventional techniques. By the success probability, we mean the probability of estimating all the tonal frequency components within an error of $1 / N$ where $N$ is the number of measurements.

We briefly summarize the notations used in this paper. Vectors are written as boldface lowercase letters (e.g., r), and matrices are denoted by boldface uppercase letters (e.g., A). For a vector $\mathbf{r} \in \mathbb{C}^{N}, \mathbf{r}[n]$ is the $n$th element of r. Toep $(\mathbf{r}) \in \mathbb{C}^{N \times N}$ is the Hermitian Toeplitz matrix with $\mathbf{r}$ as its first column. For a matrix $\mathbf{A} \in \mathbb{C}^{N \times L}, \mathbf{a}_{l} \in \mathbb{C}^{N}$ is the $l$ th column of $\mathbf{A} . \mathbf{A}^{T}$ and $\mathbf{A}^{*}$ are the transpose and the conjugate transpose of $\mathbf{A}$, respectively. $\|\mathbf{A}\|_{F}$ is the Frobenius norm of $\mathbf{A}$. For a square matrix $\mathbf{M} \in \mathbb{C}^{N \times N}, \operatorname{tr}(\mathbf{M})$ is the trace of $\mathbf{M}$.

\section{Signal model}

We consider the practical scenarios where the tonal signal dominates the received signal and is used in detecting a marine object. The signal $r(t)$ detected by the passive sonar is expressed as $[5,6,16]$

$$
r(t)=r_{\text {tonal }}(t)+r_{\text {propeller }}(t)+r_{\text {hydro }}(t)+u(t) .
$$

$r_{\text {tonal }}(t)$ is the tonal signal generated from machinery components of a marine object, and $r_{\text {propeller }}(t)$ is the propeller noise produced by the rotation of a propeller and then modulated by cavitation. $r_{\text {hydro }}(t)$ is the hydrodynamic noise caused by the friction between a marine object and waves, and $u(t)$ is the ambient noise including surface noise, molecular motions, and seismic noise. Furthermore, it is well-known that the tonal signal is expressed as a linear combination of complex sinusoids, i.e.,

$$
r_{\text {tonal }}(t)=\sum_{i=1}^{k} A_{i} \cos \left(2 \pi f_{i} t\right),
$$

where $A_{i} \in \mathbb{C}$ is the (complex) amplitude of the $i$ th frequency component [16]. Also, the propeller noise is given by [6] 


$$
r_{\text {propeller }}(t)=\left(1+\sum_{m=1}^{p} B_{m} \cos \left(2 \pi\left(m \tilde{f}_{0}\right) t\right)\right) c(t),
$$

where $\widetilde{f}_{0}$ is the undesired harmonic frequency caused by propeller rotations, $B_{m} \in \mathbb{C}$ is the amplitude of the $m$ th harmonic frequency component, and $c(t)$ is the Gaussian cavitation noise. Then, the signal $\mathbf{r}_{l} \in \mathbb{C}^{\widetilde{N}}$ sampled at the $l$ th channel can be expressed as

$$
\begin{aligned}
\mathbf{r}_{l}[n]= & \sum_{i=1}^{k} A_{i l} \cos \left(2 \pi \frac{f_{i}}{f_{s}} n\right) \\
& +\left(1+\sum_{m=1}^{p} B_{m l} \cos \left(2 \pi \frac{m \tilde{f}_{0}}{f_{s}} n\right)\right) \mathbf{c}_{l}[n] \\
& +\mathbf{v}_{l}[n],
\end{aligned}
$$

where $f_{s}$ is the sampling frequency and $\mathbf{v}_{l}$ is sum of the hydrodynamic noise and the ambient noise.

\section{Proposed method}

In this section, we describe the proposed technique to estimate the tonal frequency components $\left(f_{1}, \cdots, f_{k}\right)$ from $\mathbf{r}_{1}, \cdots, \mathbf{r}_{L}$. The proposed technique consists of two main operations: (1) pre-filtering to suppress the undesired frequency components and noise and (2) estimation of the tonal frequency components from the filtered signal using a modified ANM. We first describe the pre-filtering step and then demonstrate the tonal frequency estimation using the modified ANM.

\subsection{Noise suppression via pre-filtering}

In the pre-filtering step, we first apply the low-pass filter (LPF) followed by the decimation to the sampled signals $\left\{\mathbf{r}_{i}\right\}_{i=1}^{L}$. In order to prevent the aliasing phenomenon, we set the cutoff frequency $f_{c}$ of the LPF to be smaller than $f_{s} / \alpha$ where $\alpha$ is the decimation ratio. Since the desired tonal signal consists of low frequency components [6], we need to preserve the tonal component yet filter out unwanted high frequency interferences and noise. Using the low-pass filter with the cutoff frequency $f_{c}$ and decimation with the decimation ratio $\alpha(\alpha>1)$, the output of LPF and decimation process $\widetilde{\mathbf{r}}_{l}$ at the $l$ th channel is given by

$$
\begin{aligned}
\tilde{\mathbf{r}}_{l}[n]= & \sum_{i=1}^{k} A_{i l} \cos \left(2 \pi \frac{f_{i}}{f_{s} / \alpha} n\right)+\widetilde{\mathbf{c}}_{l}[n]+\widetilde{\mathbf{v}}_{l}[n], \\
& n=0, \cdots, N
\end{aligned}
$$

where $N$ is $\tilde{N} / \alpha, \widetilde{\mathbf{c}}_{l}[n]$ is the filtered propeller noise, and $\widetilde{\mathbf{v}}_{l}[n]$ is the sum of the filtered hydrodynamic and ambient noises.

Also, we used an auto-correlation filter defined as $R_{\mathbf{x x}}(\tau)=F^{-1}\left[|\hat{X}(f)|^{2}\right]$ where $\hat{X}(f)$ is the Fourier transform of $\mathbf{x}, F^{-1}$ is an inverse Fourier transform function, and $\tau$ is a time-lag variable. Note that an auto-correlation filter has the property of boosting the periodic signal and suppressing the uncorrelated stochastic noise [17]. Thus, using the auto-correlation filter, desired periodic tonal signal is strengthened while propeller and ambient noise components are suppressed [18]. As a result, the pre-filtered output $\mathbf{p}_{l}$ at the $l$ th channel is given by

$$
\begin{aligned}
\mathbf{p}_{l}[n] & =\sum_{i=1}^{k} A_{i l} \cos \left(2 \pi \frac{f_{i}}{f_{s} / \alpha} n\right)+\mathbf{w}_{l}[n] \\
& =\mathbf{s}_{l}[n]+\mathbf{w}_{l}[n]
\end{aligned}
$$

where

$$
\mathbf{s}_{l}[n]=\sum_{i=1}^{k} A_{i l} \cos \left(2 \pi \frac{f_{i}}{f_{s} / \alpha} n\right),
$$

and $\mathbf{w}_{l}$ is the output of the auto-correlation filter corresponding to the input $\widetilde{\mathbf{c}}_{l}+\widetilde{\mathbf{v}}_{l}$. In $[17,18]$, it has been shown that if $\widetilde{\mathbf{c}}_{l}$ and $\widetilde{\mathbf{v}}_{l}$ are white Gaussian, then the power spectrum of $\mathbf{w}_{l}$ is negligible.

Note that

$$
\frac{\mathbf{s}_{l}}{\sqrt{N}}=\sum_{i=1}^{k} \frac{A_{i l}}{2} \mathbf{a}\left(\frac{f_{i}}{f_{s} / \alpha}\right)+\sum_{i=1}^{k} \frac{A_{i l}}{2} \mathbf{a}\left(1-\frac{f_{i}}{f_{s} / \alpha}\right),
$$

where $\mathbf{a}(f)=\left[1, e^{j 2 \pi f}, \cdots, e^{j 2 \pi f(N-1)}\right]^{T} / \sqrt{N}$. Thus, $\mathbf{S}=$ $\left[\begin{array}{lll}\mathbf{s}_{1} & \cdots & \mathbf{s}_{L}\end{array}\right] \in \mathbb{C}^{N \times L}$ can be expressed as

$$
\mathbf{S}=\sum_{i=1}^{k}\left(\mathbf{a}\left(\frac{f_{i}}{f_{s} / \alpha}\right)+\mathbf{a}\left(1-\frac{f_{i}}{f_{s} / \alpha}\right)\right) \mathbf{x}_{i}^{*},
$$

where $\mathbf{x}_{i}=\frac{\sqrt{N}}{2}\left[A_{i 1} \cdots A_{i L}\right]^{*}$.

\subsection{Tonal frequency estimation via modified ANM}

The primary goal after the pre-filtering is to estimate the tonal frequency components $\left(f_{1}, \cdots, f_{k}\right)$ in $\mathbf{S}$ from the prefiltered signal $\mathbf{P}=\left[\begin{array}{lll}\mathbf{p}_{1} & \cdots & \mathbf{p}_{L}\end{array}\right]=\mathbf{S}+\mathbf{W}$. To this end, we first introduce an atom $\mathbf{A}(f, \mathbf{b})$, defined as

$$
\mathbf{A}(f, \mathbf{b})=\mathbf{a}(f) \mathbf{b}^{*} \in \mathbb{C}^{N \times L},
$$

where $f \in[0,1]$ and $\mathbf{b} \in \mathbb{C}^{L}$ is an $\ell_{2}$-normalized vector (i.e., $\|\mathbf{b}\|_{2}=1$ ). We also denote the set of atoms as

$$
\mathcal{A}=\left\{\mathbf{A}(f, \mathbf{b}): f \in[0,1], \mathbf{b} \in \mathbb{C}^{L},\|\mathbf{b}\|_{2}=1\right\} .
$$

In a nutshell, the atoms in $\mathcal{A}$ are building blocks of the desired signal $\mathbf{S}$, i.e., $\mathbf{S}$ can be expressed as an affine combination of some atoms in $\mathcal{A}$ :

$$
\begin{aligned}
\mathbf{S}= & \sum_{i=1}^{k}\left\|\mathbf{x}_{i}\right\|_{2} \mathbf{A}\left(\frac{f_{i}}{f_{s} / \alpha}, \frac{\mathbf{x}_{i}}{\left\|\mathbf{x}_{i}\right\|_{2}}\right) \\
& +\sum_{i=1}^{k}\left\|\mathbf{x}_{i}\right\|_{2} \mathbf{A}\left(1-\frac{f_{i}}{f_{s} / \alpha}, \frac{\mathbf{x}_{i}}{\left\|\mathbf{x}_{i}\right\|_{2}}\right) .
\end{aligned}
$$


One can notice that while $\mathcal{A}$ contains an infinite number of atoms, the number of atoms used in the representation (13) is very small. In this sense, we can readily say that $\mathbf{S}$ is sparse with respect to $\mathcal{A}$.

\subsubsection{Conventional ANM}

In the same vein as the $\ell_{0}$-norm minimization technique in $\mathrm{CS}$, the sparse signal $\mathbf{S}$ (with respect to $\mathcal{A}$ ) can be reconstructed from $\mathbf{P}$ by solving $[15,19]$

$$
\min _{\mathbf{X}} \frac{1}{2}\|\mathbf{X}-\mathbf{P}\|_{F}^{2}+\tau\|\mathbf{X}\|_{\mathcal{A}, 0},
$$

where $\tau(>0)$ is the pre-determined regularization parameter and $\|\mathbf{X}\|_{\mathcal{A}, 0}$ is defined as

$$
\|\mathbf{X}\|_{\mathcal{A}, 0}=\inf _{K}\left\{K: \mathbf{X}=\sum_{i=1}^{K} c_{k} \mathbf{A}_{k}, c_{k} \geq 0, \mathbf{A}_{k} \in \mathcal{A}\right\} .
$$

Finding the solution to (14 ), however, requires a combinatorial search over all possible subspaces spanned by the atoms in $\mathcal{A}$, so that direct (exhaustive search) approach is infeasible for most practical scenarios. To perform the reconstruction task, the ANM technique can be employed. The key idea of ANM is to replace the nonconvex term $\|\mathbf{X}\|_{\mathcal{A}, 0}$ in (14) with its convex surrogate atomic norm $\|\mathbf{X}\|_{\mathcal{A}}$ defined as

$$
\|\mathbf{X}\|_{\mathcal{A}}=\inf \left\{\sum_{k} c_{k}: \mathbf{X}=\sum_{k} c_{k} \mathbf{A}_{k}, c_{k} \geq 0, \mathbf{A}_{k} \in \mathcal{A}\right\} .
$$

In other words, ANM reconstructs $\mathbf{S}$ by solving [15]

$$
\min _{\mathbf{X}} \frac{1}{2}\|\mathbf{X}-\mathbf{P}\|_{F}^{2}+\tau\|\mathbf{X}\|_{\mathcal{A}} \text {. }
$$

By using [15, Theorem 1]

$$
\begin{aligned}
\|\mathbf{X}\|_{\mathcal{A}}=\inf & \left\{\frac{1}{2}(\operatorname{tr}(\mathbf{W})+\operatorname{tr}(\operatorname{Toep}(\mathbf{u})))\right. \\
: & {\left.\left[\begin{array}{ll}
\mathbf{W} & \mathbf{X}^{*} \\
\mathbf{X} & \operatorname{Toep}(\mathbf{u})
\end{array}\right] \succeq 0\right\}, }
\end{aligned}
$$

and thus we obtain the equivalent form of (17) as

$$
\begin{array}{ll}
\min _{\mathbf{X}, \mathbf{W}, \mathbf{u}} & \|\mathbf{X}-\mathbf{P}\|_{F}^{2}+\tau(\operatorname{tr}(\mathbf{W})+\operatorname{tr}(\operatorname{Toep}(\mathbf{u}))) \\
\text { s.t. } & {\left[\begin{array}{ll}
\mathbf{W} & \mathbf{X}^{*} \\
\mathbf{X} & \operatorname{Toep}(\mathbf{u})
\end{array}\right] \succeq 0}
\end{array}
$$

Note that the problem (19) can be solved effectively via a well-known SDP solver (e.g., SDPT3 [19]). Note also that since the computational complexity of the SDP is $\mathcal{O}\left(M^{5 / 2}\right)[20]$ and the number of samples $M$ is reduced to $N=\widetilde{N} / \alpha$ by the decimation process, the computation complexity of the estimation step is marginal.

We now present a method to estimate the frequency components $\left(f_{1}, \cdots, f_{k}\right)$ in $\mathbf{S}$ (see (13)). To this end, we first derive a dual problem of (17). By introducing a new variable $\mathbf{Y}=\mathbf{X}$ for the problem (17), the Lagrangian $L(\mathbf{X}, \mathbf{Y}, \mathbf{Z})$ associated with (17) is expressed as

$$
\begin{aligned}
L(\mathbf{X}, \mathbf{Y}, \mathbf{Z}) & \\
= & \frac{1}{2}\|\mathbf{X}-\mathbf{P}\|_{F}^{2}+\tau\|\mathbf{Y}\|_{\mathcal{A}}+\operatorname{Re}\left(\operatorname{tr}\left(\mathbf{Z}^{*}(\mathbf{X}-\mathbf{Y})\right)\right) \\
= & \left(\frac{1}{2}\|\mathbf{X}-\mathbf{P}\|_{F}^{2}+\operatorname{Re}\left(\operatorname{tr}\left(\mathbf{Z}^{*} \mathbf{X}\right)\right)\right) \\
& +\left(\tau\|\mathbf{Y}\|_{\mathcal{A}}-\operatorname{Re}\left(\operatorname{tr}\left(\mathbf{Z}^{*} \mathbf{Y}\right)\right)\right),
\end{aligned}
$$

where $\mathbf{Z}$ is the dual variable. One can easily show that

$$
\begin{gathered}
\min _{\mathbf{X}} \frac{1}{2}\|\mathbf{X}-\mathbf{P}\|_{F}^{2}+\operatorname{Re}\left(\operatorname{tr}\left(\mathbf{Z}^{*} \mathbf{X}\right)\right) \\
=\frac{1}{2}\left(\|\mathbf{P}\|_{F}^{2}-\|\mathbf{P}-\mathbf{Z}\|_{F}^{2}\right)
\end{gathered}
$$

and

$$
\begin{aligned}
& \min _{\mathbf{Y}} \tau\|\mathbf{Y}\|_{\mathcal{A}}-\operatorname{Re}\left(\operatorname{tr}\left(\mathbf{Z}^{*} \mathbf{Y}\right)\right) \\
& \quad=\min _{\mathbf{Y}}\|\mathbf{Y}\|_{\mathcal{A}}\left(\tau-\operatorname{Re}\left(\operatorname{tr}\left(\mathbf{Z}^{*} \mathbf{Y} /\|\mathbf{Y}\|_{\mathcal{A}}\right)\right)\right) \\
& \quad= \begin{cases}0 & \|\mathbf{Z}\|_{\mathcal{A}}^{*} \leq \tau \\
-\infty & \|\mathbf{Z}\|_{\mathcal{A}}^{*}>\tau,\end{cases}
\end{aligned}
$$

where $\|\mathbf{Z}\|_{\mathcal{A}}^{*}$ is the dual norm of the atomic norm defined as $\|\mathbf{Z}\|_{\mathcal{A}}^{*}=\sup _{\|\mathbf{X}\|_{\mathcal{A}} \leq 1} \operatorname{Re}\left(\operatorname{tr}\left(\mathbf{X}^{*} \mathbf{Z}\right)\right)$. From (20)-(22), the dual problem of (17) is given by

$$
\begin{array}{ll}
\max _{\mathbf{Z}} & \frac{1}{2}\left(\|\mathbf{P}\|_{F}^{2}-\|\mathbf{P}-\mathbf{Z}\|_{F}^{2}\right) \\
\text { s.t. } & \|\mathbf{Z}\|_{\mathcal{A}}^{*} \leq \tau .
\end{array}
$$

Let $\widehat{\mathbf{Z}}$ be the solution to (23). Then, the correlation $Q(f)=$ $\left\|\widehat{\mathbf{Z}}^{*} \mathbf{a}(f)\right\|_{2}$ between the solution $\widehat{\mathbf{Z}}$ and the atom $\mathbf{a}(f)$ is maximized when $f$ is one of the tonal frequency components (i.e., $\left.f \in\left\{f_{1}, \cdots, f_{k}\right\}\right)$. Specifically, $Q(f)$ satisfies [15]

$$
\left\{\begin{array}{l}
Q(f)=\tau, \text { if } f \in\left\{f_{1}, \cdots, f_{k}\right\} \\
Q(f)<\tau, \text { if } f \notin\left\{f_{1}, \cdots, f_{k}\right\}
\end{array} .\right.
$$

Therefore, we can estimate the desired frequency components $\left(f_{1}, \cdots, f_{k}\right)$ by solving $Q(f)=\tau$.

\subsubsection{Modified ANM}

One potential limitation of the conventional ANM technique is that the performance is not so appealing when the source vectors $\mathbf{s}_{1}, \cdots, \mathbf{s}_{L}$ are strongly correlated. For better understanding of this issue, we take the following example. If the correlation between $\mathbf{s}_{1}$ and $\mathbf{s}_{2}$ is extremely strong (i.e., $\frac{\left|\left\langle\mathbf{s}_{1}, \mathbf{s}_{2}\right\rangle\right|}{\left\|\mathbf{s}_{1}\right\|_{2}\left\|\mathbf{s}_{2}\right\|_{2}} \approx 1$ ), then $\mathbf{s}_{1} \approx \mathbf{s}_{2}$ (up to a constant factor $^{1}$ ) and therefore the corresponding measurement vectors $\mathbf{p}_{1}$ and $\mathbf{p}_{2}$ would be also similar (i.e., $\mathbf{p}_{1} \approx \mathbf{p}_{2}$ ). As a result, ANM cannot obtain any additional information on ${ }^{1}$ Note that $\frac{\left|\left\langle\mathbf{s}_{1}, \mathbf{s}_{2}\right\rangle\right|}{\left\|\mathbf{s}_{1}\right\|_{2}\left\|\mathbf{s}_{2}\right\|_{2}} \leq 1$ and the equality is attained if and only if
$\mathbf{s}_{1} /\left\|\mathbf{s}_{1}\right\|_{2}=\mathbf{s}_{2} /\left\|\mathbf{s}_{2}\right\|_{2}$. 
the frequency components from $\mathbf{p}_{2}$, which in turn implies that there is no benefit of using multiple measurement vectors $\mathbf{p}_{1}$ and $\mathbf{p}_{2}$.

In order to achieve reliable performance even when the source vectors are highly correlated, we exploit an orthonormal basis of the measurement space in estimating the frequency components. By the measurement space, we mean the subspace spanned by the measurement vectors $\mathbf{p}_{1}, \cdots, \mathbf{p}_{L}$. Specifically, we replace the measurement matrix $\mathbf{P}$ in (23) with the orthonormal basis $\mathbf{U}$ of $\operatorname{span}(\mathbf{P})$. Suppose the observation matrix $\mathbf{P} \in \mathbb{R}^{N \times L}$ has full column rank (i.e., $\operatorname{rank}(P)=L$ ), then an orthonormal basis of the measurement space can be obtained by performing an singular value decomposition (SVD) operation on $\mathbf{P}$. Specifically, if $\mathbf{P}=\mathbf{U} \boldsymbol{\Sigma} \mathbf{V}^{H}$ where $\mathbf{U} \in \mathbb{R}^{N \times L}$, $\boldsymbol{\Sigma} \in \mathbb{R}^{L \times L}$, and $\mathbf{V} \in \mathbb{R}^{L \times L}$, then $\mathbf{U}$ is an orthonormal basis of $\operatorname{span}(\mathbf{P})$. After obtaining the orthonormal basis $\mathbf{U}$, we reformulate (23) as:

$$
\begin{array}{cl}
\max _{\mathbf{Z}} & \frac{1}{2}\left(\|\mathbf{U}\|_{F}^{2}-\|\mathbf{U}-\mathbf{Z}\|_{F}^{2}\right) \\
\text { s.t. } & \|\mathbf{Z}\|_{\mathcal{A}}^{*} \leq \tau
\end{array}
$$

Let $\widetilde{\mathbf{Z}}$ be the solution to (25). Then, the proposed technique estimates the desired tonal frequency components $\left(f_{1}, \cdots, f_{k}\right)$ by solving

$$
Q(f)=\left\|\widetilde{\mathbf{Z}}^{*} \mathbf{a}(f)\right\|_{2}=\tau .
$$

We depict the overall block diagram of the proposed scheme in Fig. 2. To verify the performance of the modified ANM technique, we perform the numerical simulation in the scenario where the source vectors are highly correlated. In our simulation, we generate the strongly correlated source vectors $\mathbf{s}_{1}, \mathbf{s}_{2}$ such that $\left|\left\langle\mathbf{s}_{1}, \mathbf{s}_{2}\right\rangle\right| \approx 0.99$, and each source vector consists of $k$ tonal frequency components. As a metric to evaluate the performance of each technique, we employ the exact reconstruction ratio (ERR) defined as

$$
E R R=\frac{\text { Number of successful trials }}{\text { Number of trials }}
$$

Here, each trial is defined to be successful if all the estimated frequencies $\widehat{f}_{i}$ satisfy $\left|f_{i}-\widehat{f}_{i}\right|<\frac{1}{2 N}$ where $f_{i}$ is the $i$ th target frequency component and $N$ is the number of measurements. Note that we perform 2000 independent trials for each point of the technique. In Fig. 3, we plot the ERR performance of each technique as a function of $k$. Overall, the ERR performance improves with the number of measurement vectors $L$. One can see from Fig. 3 that the performance improvement of the modified ANM technique performs much better than that of the conventional ANM method. The key reason for this result is that $\mathbf{p}_{1} \approx$ $\mathbf{p}_{2}$ and thus the conventional ANM obtains little information on the frequency components from $\mathbf{p}_{2}$. Additionally, one can observe that the ERR performance of the modified ANM is the same as that of the conventional ANM when $L=1$, since the orthonormal basis of the measurement space coincides with the measurement vector (up to a constant factor) in this case. In Table 1, we summarize the critical sparsities of the proposed scheme and the conventional ANM technique. By the critical sparsity, we mean the maximum number of frequency components at which ERR is above 0.9. From the table, we can observe that our scheme outperforms the conventional ANM technique for all $L$. For example, when $L=5$, the critical sparsity of the proposed scheme is three times higher than that obtained by the conventional ANM technique.

\section{Simulation results and discussion}

In this section, we study the performance of the proposed frequency estimation technique using both synthetic and real datasets.

\subsection{Simulation setup}

First, we test the performance of the proposed technique using synthetic data. In our simulations, we generate the tonal signal whose frequency components are uniformly

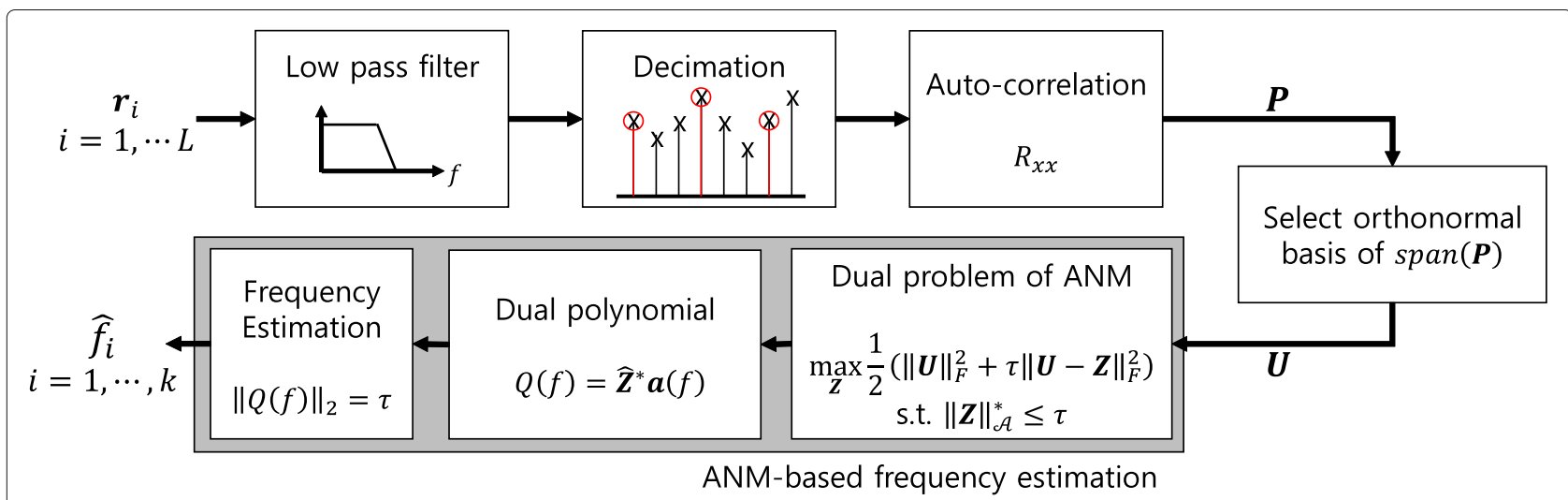

Fig. 2 Block diagram of the passive sonar systems using proposed frequency estimation scheme 


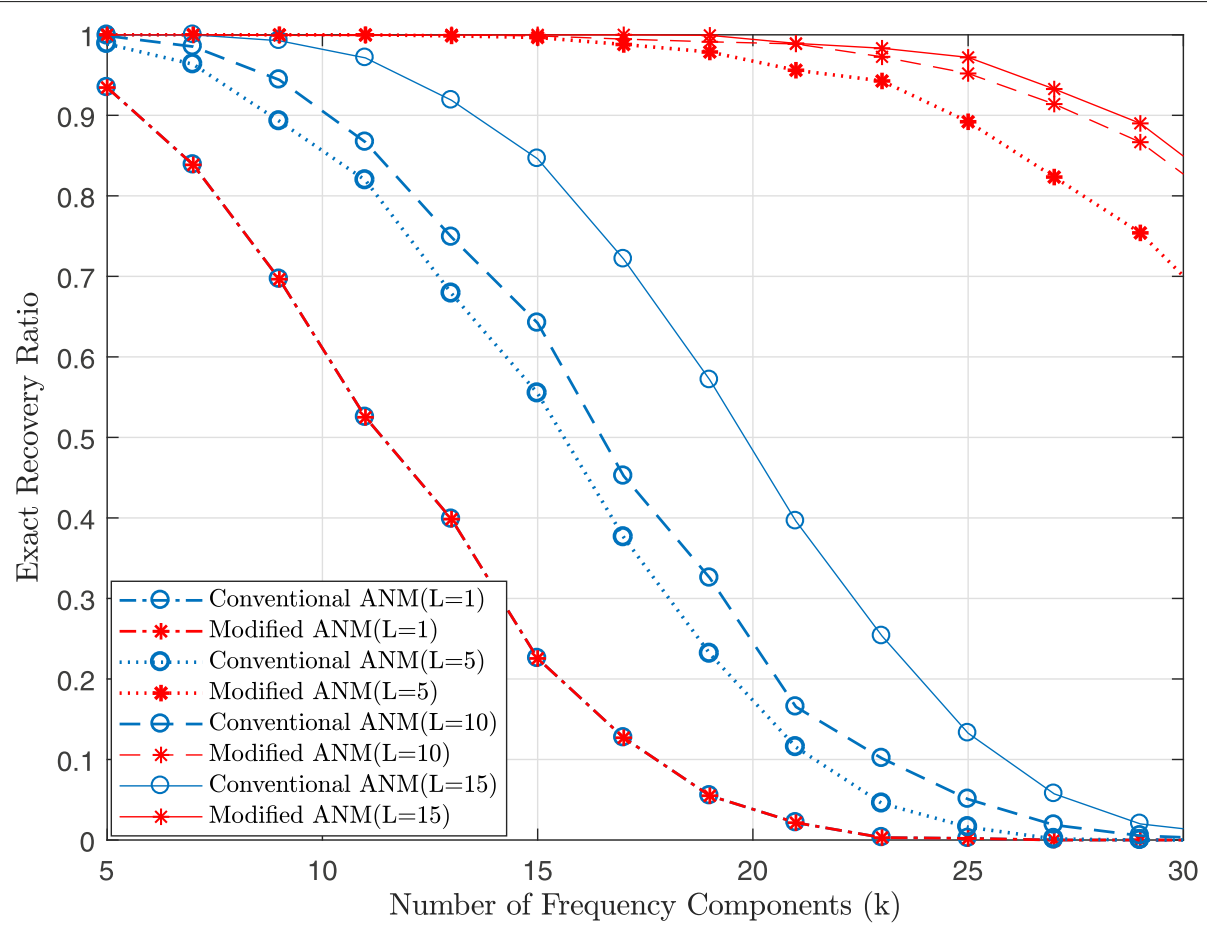

Fig. 3 The ERR performance of the conventional and modified ANM techniques when the source vectors are highly correlated

chosen at random in the range of $0-500 \mathrm{~Hz}$ and amplitudes of frequency components are drawn i.i.d. from a Gaussian distribution $\mathcal{N}(1,0.1)$. The base frequency $\widetilde{f}_{0}$ of the propeller noise is set to $10 \mathrm{~Hz}$, and we assume the scenario where the propeller noise consists of three harmonic components (i.e., $p=3$ in (4)). The other noise component $\mathbf{v}_{l}$ in (4) is generated as a white Gaussian with mean zero and variance $\sigma^{2}$. The sampling frequency $f_{s}$ is set to $10 \mathrm{kHz}$. In the pre-filtering step, we set the cutoff frequency of the LPF to $500 \mathrm{~Hz}$ and the decimation ratio $\alpha$ to 10 . In our simulations, the following frequency estimation techniques are considered and we perform 5000 independent trials for each point of the technique:

1) Root-MUSIC [8]

2) ESPRIT [9]

3) Matrix pencil [10]

4) Conventional ANM [19]

5) Modified ANM

We next verify the performance of the proposed technique using real data obtained from the shallow water

Table 1 Critical sparsity performance comparison of modified ANM and conventional ANM

\begin{tabular}{lllll}
\hline & $L=1$ & $L=5$ & $L=10$ & $L=15$ \\
\hline Modified ANM & 5 & 24 & 27 & 28 \\
Conventional ANM & 5 & 8 & 10 & 13 \\
\hline
\end{tabular}

evaluation cell experiment 96 (SWellEx-96). Note that the SWellEx-96 was conducted $12 \mathrm{~km}$ off the coast of San Diego, CA, in May 1996, and the target source signal consists of 13 frequency components $(49,64,79,94,112,130$, $148,166,201,235,283,338$, and $388 \mathrm{~Hz}$ ). In our simulations, we divide 4000 measurement vectors into 200 groups (20 measurement vectors in each group). Using each group of measurement vectors, we estimate the tonal frequency components.

\subsection{Results and discussion}

In Fig. 4, the ERR performance of each technique is plotted as a function of $k$ (number of desired tonal frequency components). This simulation is performed in the scenario where the signal-to-noise ratio (SNR) is SNR = $10 \log \left(\left(\sum_{i=1}^{k}\left|A_{i}\right|^{2}\right) /\left(\sum_{m=1}^{p}\left|B_{m}\right|^{2}+\sigma^{2}\right)\right)=10 \mathrm{~dB}$. As shown in Fig. 4, the ERR performance of the proposed technique is better than those of the other frequency estimation techniques in the whole range of $k$ by a large margin.

In Fig. 5, we plot the mean square error (MSE) performance of conventional ANM method and modified ANM as a function of $k$. The MSE is defined as

$$
\operatorname{MSE}=\frac{1}{k} \sum_{i=1}^{k}\left(\widehat{f}_{i}-f_{i}\right)^{2} .
$$




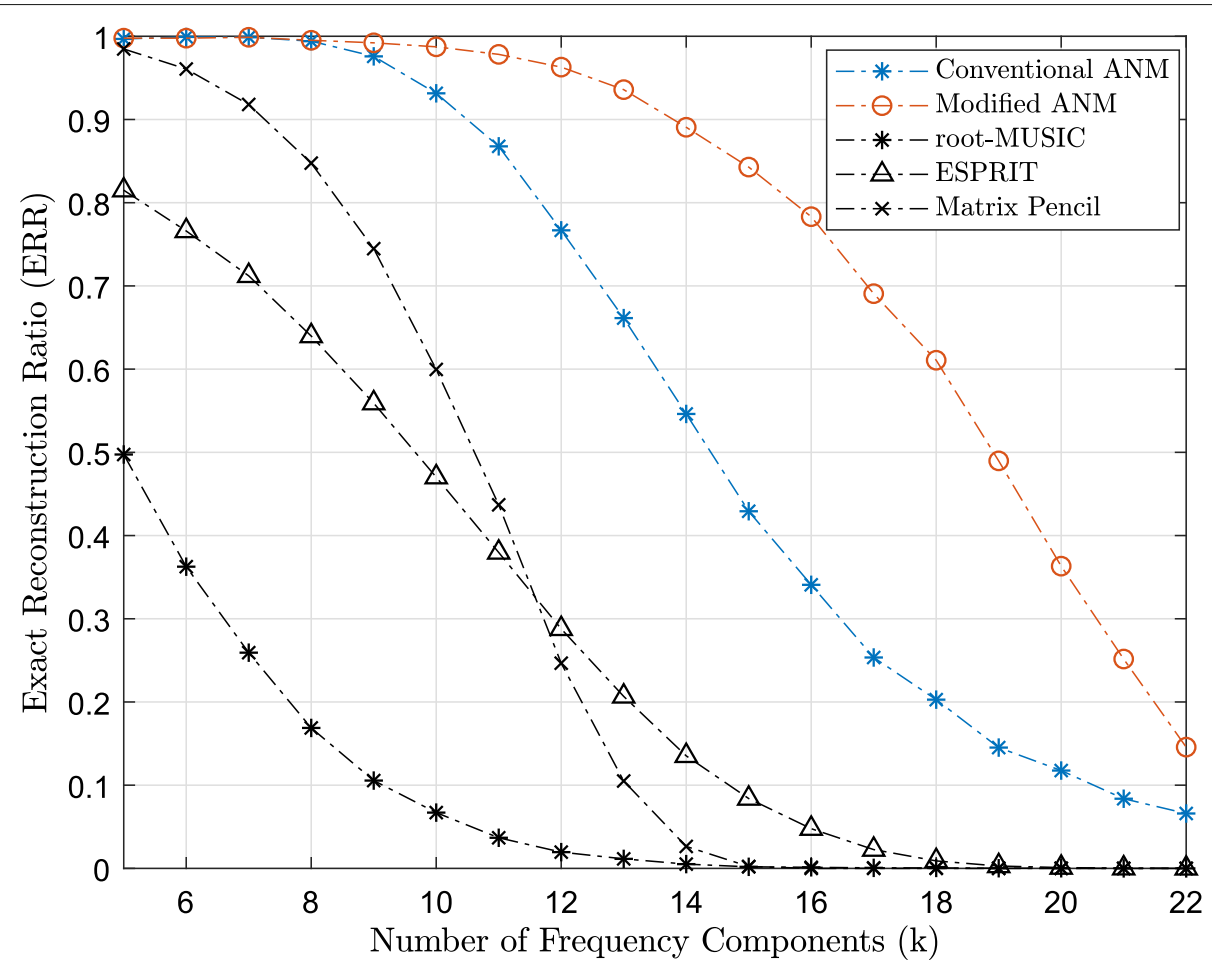

Fig. 4 ERR performance as a function of $k$

Note that the MSE performance shows how close each of the estimated frequency components is to the original frequency components. We measured the MSE only in the case where the estimation was successful. As depicted in Fig. 5, the modified ANM performs better than the conventional ANM.
In Fig. 6, we plot the MSE performance of the proposed method and other line spectrum estimation methods as a function of SNR. As shown in Fig. 6a, ANMbased algorithms outperform conventional line spectrum methods in all SNR region. In particular, when SNR = $0 \mathrm{~dB}$, the MSE of the proposed scheme is 1.5 times

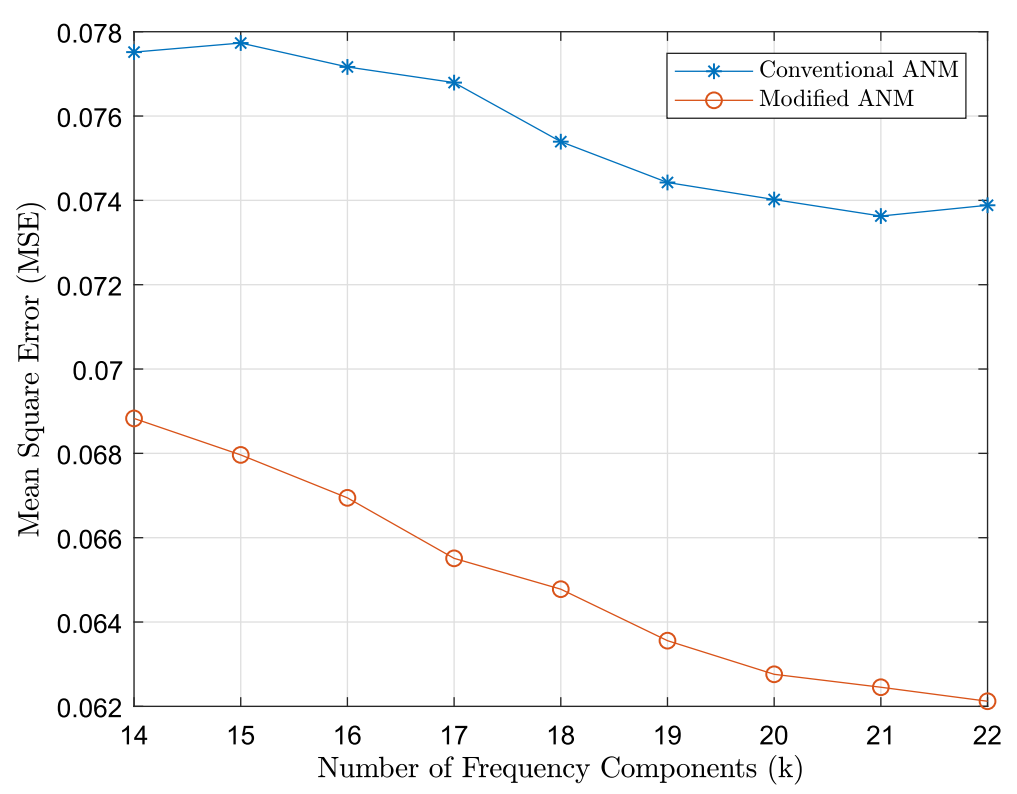

Fig. 5 MSE performance as a function of $k$ 
lower than that of the conventional ANM technique (see Fig. 6b).

In our real experiment test, we say that the $i$ th frequency component $f_{i}$ is successfully estimated if $\left|f_{i}-\widehat{f}_{i}\right|<5$. As a metric to measure the performance of the proposed technique, we exploit the success ratio $r_{\text {success }}$ and the mean square error $\mathrm{MSE}_{\text {success }}$ of the successfully estimated frequency components. Let $J$ be the index set of successfully estimated frequency components. Then, $r_{\text {success }}$ and MSE $_{\text {success }}$ are defined as

$$
\begin{aligned}
& r_{\text {success }}=\frac{|J|}{13}, \\
& \operatorname{MSE}_{\text {success }}=\frac{1}{|J|} \sum_{j \in J}\left|f_{j}-\widehat{f}_{j}\right|^{2} .
\end{aligned}
$$

In Table 2, we summarize the performance of the conventional ANM-based method and the proposed technique. One can see that the success ratio $r_{\text {success }}$ of the proposed technique is roughly 2.5 times higher than that of the conventional ANM-based technique. Furthermore,
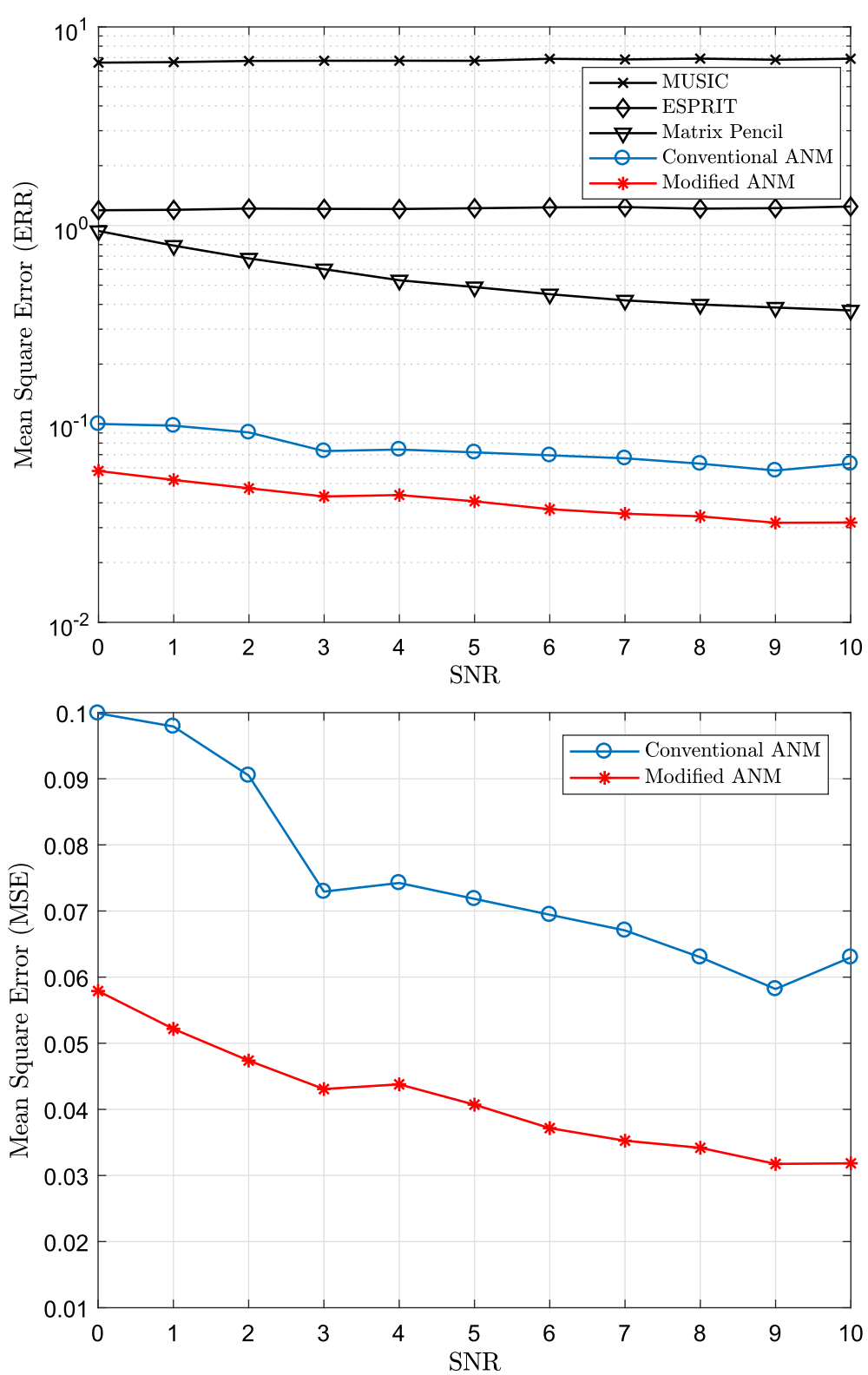

Fig. 6 MSE performance as a function of SNR. a MSE performance comparison of line spectrum estimation techniques as a function of SNR. b MSE performance comparison of ANM-based scheme as a function of SNR 
Table 2 Performance comparison of ANM-based algorithms

\begin{tabular}{lll}
\hline & Conventional ANM & Proposed ANM \\
\hline$r_{\text {success }}$ & 0.2871 & 0.6557 \\
MSE $_{\text {success }}(\mathrm{Hz})$ & 7.7159 & 6.3799 \\
\hline
\end{tabular}

the $\mathrm{MSE}_{\text {success }}$ of $\left\{\widehat{f}_{j}: j \in J\right\}$ obtained by the proposed method is 1.2 times smaller than that acquired by the conventional method.

\section{Conclusion}

In this paper, we proposed an ANM-based frequency estimation technique to identify the tonal frequency components in the passive sonar system. The proposed technique consists of two major steps. In the first step, we applied the LPF, decimation, and the auto-correlation filter to the signal detected by the passive sonar, filtering out the unwanted propeller noise and high-frequency interferences. In the second step, we formulated the tonal frequency estimation problem as an ANM problem and then estimated the desired frequency components using an orthonormal basis of the measurement space. In doing so, we could achieve reliable performance even when the source vectors are strongly correlated. Finally, using both synthetic and real datasets, we demonstrated that the proposed technique is effective in estimating the tonal frequency components.

\section{Abbreviations \\ ANM: Atomic norm minimization; CS: Compressed sensing; DFT: Discrete Fourier transform; ERR: Exact reconstruction ratio; ESPRIT: Estimation of signal parameters via rotational invariance techniques; LPF: Low-pass filter; MSE: Mean square error; MUSIC: Multiple signal classification; SDP: Semi-definite programming}

\section{Acknowledgements}

The authors would like to thank the Agency for Defense Development (ADD) in Korea for supporting this work (contract No. UD190005DD).

\section{Authors' contributions}

All the authors have participated in writing the manuscript. All authors read and approved the manuscript.

\section{Funding}

This work was supported by the Agency for Defense Development (ADD) in Korea under the contract No. UD190005DD.

\section{Availability of data and materials}

Please contact the author for synthetic data request, and the SWellEx-96 dataset used in this work is available at http://swellex96.ucsd.edu/s5.htm.

\section{Consent for publication}

Not applicable.

\section{Competing interests}

The authors declare that they have no competing interests.

\section{Author details}

${ }^{1}$ Dept. of Electrical and Computer Engineering, Seoul National Univ., 1 Gwanak-ro, Gwanak-gu, 08826 Seoul, South Korea. ${ }^{2}$ Dept. of Defense Systems Engineering, Sejong Univ., 209 Neungdong-ro, Gunja-dong, Gwangjin-gu, 05006 Seoul, South Korea.
Received: 14 December 2018 Accepted: 26 August 2019

Published online: 09 September 2019

\section{References}

1. M. S. Allahparast, S. Allahparast, C. Laschi, in 2016 Indian Control Conference (ICC). An improved tracking algorithm for underwater vessels using the passive sonar (IEEE, 2016), pp. 426-431. https://doi.org/10.1109/indiancc. 2016.7441169

2. T. Fortmann, Y. Bar-Shalom, M. Scheffe, Sonar tracking of multiple targets using joint probabilistic data association. IEEE J. Ocean. Eng. 8(3), 173-184 (1983)

3. T. C. Bailey, T. Sapatinas, K. J. Powell, W. J. Krzanowski, Signal detection in underwater sound using wavelets. J. Am. Statistical Assoc. 93(441), 73-83 (1998)

4. N. De Moura, J. De Seixas, R. Ramos, in Sonar Systems. Passive sonar signal detection and classification based on independent component analysis (InTech, 2011), pp. 93-104. https://doi.org/10.5772/18286

5. R. J. Urick, Principles of underwater sound-2. (McGraw-Hill, New York, 1975)

6. H. Cho, J. Lim, B. Ku, M. Cheong, I. Seo, H. Ko, W. Hong, Underwater radiated signal analysis in the modulation spectrogram domain. Commun. Comput. Sci. IEICE Trans. Fundam. Electron. 98(8), 1751-1759 (2015)

7. C. R. Wan, J. T. Goh, H. T. Chee, Optimal tonal detectors based on the power spectrum. IEEE J. Ocean. Eng. 25(4), 540-552 (2000)

8. A. Barabell, in Proc. IEEE Int. Conf. Acoust., Speech, Signal Process. Improving the resolution performance of eigenstructure-based direction-finding algorithms (IEEE, Boston, 1983), pp. 336-339. https://doi.org/10.1109/ icassp.1983.1172124

9. R. Roy, T. Kailath, Esprit-estimation of signal parameters via rotational invariance techniques. IEEE Trans. Acoust., Speech, Signal Process. 37(7), 984-995 (1989)

10. Y. Hua, T. K. Sarkar, Matrix pencil method for estimating parameters of exponentially damped/undamped sinusoids in noise. IEEE Trans. Acoust. Speech Signal Process. 38(5), 814-824 (1990)

11. E. J. Candès, J. Romberg, T. Tao, Robust uncertainty principles: Exact signal reconstruction from highly incomplete frequency information. IEEE Trans. Inform. Theory. 52(2), 489-509 (2006)

12. J. W. Choi, B. Shim, Y. Ding, B. Rao, D. I. Kim, Compressed sensing for wireless communications: Useful tips and tricks. IEEE Commun. Surv Tuts. 19(3), 1527-1550 (2017)

13. G. Tang, B. N. Bhaskar, P. Shah, B. Recht, Compressed sensing off the grid. IEEE Trans. Inform. Theory. 59(11), 7465-7490 (2013)

14. B. N. Bhaskar, G. Tang, B. Recht, Atomic norm denoising with applications to line spectral estimation. IEEE Trans. Signal Process. 61(23), 5987-5999 (2013)

15. Y. Li, Y. Chi, Off-the-grid line spectrum denoising and estimation with multiple measurement vectors. IEEE Trans. Signal Process. 64(5), 1257-1269 (2016)

16. M. Zeytinoglu, K. M. Wong, Detection of harmonic sets. IEEE Trans. Signal Process. 43(11), 2618-2630 (1995)

17. G. Luo, D. Osypiw, M. Irle, Real-time condition monitoring by significant and natural frequencies analysis of vibration signal with wavelet filter and autocorrelation enhancement. J. Sound Vib. 236(3), 413-430 (2000)

18. W. Su, F. Wang, H. Zhu, Z. Zhang, Z. Guo, Rolling element bearing faults diagnosis based on optimal Morlet wavelet filter and autocorrelation enhancement. Mech. Syst. Signal Process. 24(5), 1458-1472 (2010)

19. Z. Yang, L. Xie, Exact joint sparse frequency recovery via optimization methods. IEEE Trans. Signal Process. 64(3), 5145-5157 (2016)

20. F. A. Potra, S. J. Wright, Interior-point methods. J. Computat. Appl. Math. 124(1), 281-302 (2000)

\section{Publisher's Note}

Springer Nature remains neutral with regard to jurisdictional claims in published maps and institutional affiliations. 